\section{Monika Simon}

HEG Lucerne - Lucerne University of Applied

Sciences and Arts

Suisse

monika.simon@hslu.ch
UDK 811.133.1'243:659.1

DOI: 10.4312/vestnik.11.251-269

\title{
CULTURE(S) PUBLICITAIRE(S) EN CLASSE DE FRANÇAIS LANGUE ÉTRANGĖRE : " FRANCE IS IN THE AIR », ANALYSE D'UNE CAMPAGNE
}

\section{INTRODUCTION}

Omniprésente dans notre quotidien, la publicité apparaît à la fois comme le lieu de construction et le reflet de valeurs sociétales propres au contexte dans lequel elle s'inscrit. En tant que telle, elle propose des contenus et des scénarios caractéristiques, des 'artefacts' qui se prêtent idéalement à une sensibilisation pour l'altérité culturelle.

Dans le cas de l'enseignement du FLE (français langue étrangère), elle se présente comme un outil particulièrement riche pour faire découvrir aux étudiants la culture française sous diverses facettes. Par le biais, tout d'abord, de stéréotypes, clichés et préjugés largement répandus et si familiers (marinières, baguettes, savoir-vivre, ...), elle offre aux apprenants un accès aussi facile que plaisant au phénomène. Par-delà, la culture publicitaire française, qui, davantage que toute autre, démultiplie les références nationales, incite aussi ses lecteurs à déceler dans ses productions et messages la représentation d'une 'francité' réelle ou fantasmée. Enfin, figure d'exception en termes de créativité, la production publicitaire française présente des caractéristiques stylistiques dont le repérage et l'analyse permettra aux apprenants de découvrir les traits saillants d'une communication particulière, soucieuse à la fois du commun et du plus singulier.

Cet article se penchera d'abord sur des considérations théoriques d'un phénomène au carrefour entre commerce, culture et société. Dans ce contexte, il s'arrêtera notamment à l'approche sémiotique et sociosémiotique, qui envisage le discours publicitaire comme un espace où sont générés les phénomènes de société, un lieu de construction d'une réalité culturelle et sociale. En un second temps, il cherchera à définir en quoi consiste l'« exception française » en matière de communication publicitaire en la considérant dans une perspective comparative internationale. Pour finir, il procédera à l'analyse de quelques 'artefacts' publicitaires de la dernière campagne d'Air France intitulée « France is in the Air », dans le but de déterminer dans quelle mesure certains motifs typiques et récurrents 
reflètent une réalité tangible ou renvoient plutôt à des représentations factices et idéalisées de la France contemporaine.

\section{CONSIDÉRATIONS THÉORIQUES : LA PUBLICITÉ COMME MIROIR SOCIO-CULTUREL}

Avant de nous pencher sur le phénomène, arrêtons-nous d'abord sur le terme qui le désigne. Attesté pour la première fois en 1689, le mot 'publicité' est initialement employé dans le langage juridique pour désigner " l'action de porter à la connaissance du public", donc de propager une information pour la rendre accessible au plus grand nombre de personnes (Rey 2010, 1800). Vers la moitié du XIX ${ }^{\mathrm{e}}$ siècle, avec le développement de la presse à grand tirage, le terme entre dans le champ sémantique du commercial, dans lequel il se substituera progressivement au terme de 'réclame' pour désigner le fait de mettre en scène des marchandises dans l'espace public, de les valoriser à des fins commerciales. Une telle évolution l'amènera à se revendiquer, un siècle plus tard, une reconnaissance professionnelle : « la publicité, ce n'est plus la réclame mais une profession qui exige une dose de psychologie, qui met en œuvre des moyens techniques et des vertus créatives, qui utilise des méthodes d'organisation pour lesquelles le publicitaire n'a rien à envier aux autres professionnels " (Fédération française de la publicité 1956, citée dans De Iulio 2016, 6). Au fil du temps, la notion a pu ainsi se constituer un réseau de significations très large, recouvrant de manière générique l'ensemble des techniques de communication mises en œuvre pour « exercer une action sur le public à des fins commerciales » (Rey 2010, 1800), pour lui faire connaître et valoriser un produit dans une visée marchande. S'il existe aujourd'hui une pléthore de définitions (les conceptions du terme varient en fonction de l'ancrage professionnel de son auteur), la plasticité de cette notion - qui recoupe à la fois la finalité de l'action (persuasion commerciale), le processus de mise en œuvre, le cheminement créatif et son résultat (produit publicitaire) - représente, pour le chercheur, à la fois une richesse à exploiter et un écueil. Un premier défi consistera donc à assigner à cet objet d'étude « en mouvance perpétuelle » (De Iulio 2016, 9) des frontières méthodologiques.

Phénomène complexe lié à des enjeux divers, la publicité a donné lieu à de nombreuses recherches issues de perspectives et de disciplines très variées. Ce n'est véritablement qu'à partir de la moitié du XX $\mathrm{XX}^{\mathrm{e}}$ siècle qu'elle s'établit comme objet d'étude. Dans un contexte marqué à la fois par l'extension des médias et le développement, en Europe, des propagandes totalitaires, l'intérêt des chercheurs se porte tout d'abord sur l'influence qu'elle exerce sur le comportement des consommateurs (Sacriste 2001, 488). Les études réalisées dans le domaine notamment de la psychologie sociale concentrent leur attention surtout sur les variables, les 'stimuli' qui déterminent l'attitude vis-à-vis des produits et l'acte d'achat. La publicité, dans ce contexte, est assimilée à une communication de 
masse dont l'objectif est de pousser les individus à l'acquisition de biens en adoptant les représentations sociales de la consommation qui leurs sont liées. L'attention, à ce stade, s'arrête au pouvoir de la publicité, à la nature de son influence, mais pas à la signification qu'elle revêt pour ses destinataires. En d'autres termes, cette première phase n'envisage pas encore le rapport d'interaction entre le manifeste publicitaire et les représentations sociales et culturelles qu'entretient un certain type de société.

Dès le milieu des années 1950, les travaux de Roland Barthes ouvrent une nouvelle perspective à l'étude de la communication publicitaire en faisant entrer celle-ci dans le champ de la sémiotique. La multiplication d'« idées en forme » convie en effet à porter un regard différent sur le phénomène, comme le justifie Barthes dans la célèbre postface de ses Mythologies $^{1}$ : « Le développement de la publicité, de la grande presse, de la radio, de l'illustration, sans parler de la survivance d'une infinité de rites communicatifs (...) rend plus urgente que jamais la constitution d'une science sémiologique » (Barthes 1957, 197). À l'encontre de l'approche précédente, qui portait son attention sur les effets et donc le pouvoir implicite de la publicité dans la société, Barthes souligne que le discours publicitaire ne fait aucun mystère de sa finalité persuasive : « en publicité, la signification (...) est assurément intentionnelle » (Barthes 1964, 40). En tant que tel, il invite le chercheur à dépasser l'analyse de sa visée marchande pour porter l'attention sur le système de signification qui sous-tend sa démarche : « il faut adopter une position immanente à l'objet que l'on veut étudier, c'est-à-dire abandonner volontairement toute observation relative à l'émission ou à la réception du message, et se placer au niveau du message lui-même » (Barthes 1963, 243). Par-delà l'incitation à acheter, le message publicitaire, tel que le conçoit Roland Barthes, nous ouvre ainsi à « une représentation parlée du monde », à une « expérience de l'esprit » dans le sens où il relie son lecteur à la plus grande quantité d'expérience (de « monde ») possible, qu'il « dit » et « raconte » les « grands thèmes humains » (ibid.). Dans cette perspective, la publicité peut se lire comme le reflet de représentations collectives, elle apparaît à la fois comme le miroir et le creuset de significations et de valeurs partagées, des « mythologies » d'une société.

L'approche sémiologique de Roland Barthes trouve un prolongement dans l'œuvre de Umberto Eco qui davantage encore que ce dernier s'attache à démontrer l'ancrage culturel et social de la publicité. Dans La structure absente, l'auteur met en évidence que tout processus de communication est sous-tendu par une règle ou un code reposant sur des conventions établies dans la société, sur un « déjà acquis » de " solutions codifiées » (Eco 1972, 257). Cautionnant le système de normes et valeurs du cadre dans lequel elle intervient, elle permet d'analyser la situation d'une société dans son ensemble, comme un système préconstitué, qu'elle contribue à confirmer et à reproduire.

Les études de Roland Barthes et d'Umberto Eco marquent un tournant décisif en ouvrant de nouvelles pistes à l'analyse du discours publicitaire. Établissant celle-ci au-delà

1 « Le mythe aujourd'hui ». 
de l'approche commerciale, leur démarche vise à comprendre comment la communication marchande constitue un système signifiant destiné à promouvoir et à renforcer une forme de pensée dominante. L'approche sémiotique inaugurée par ces deux auteurs exercera une influence considérable sur la manière de penser le rôle de la publicité dans la société ; dans cette perspective, cependant, l'analyse des signes publicitaires se configure, dans un premier temps, comme une critique éthico-idéologique de leur fonctionnement (De Iulio 2016, 23). Il faudra attendre le tournant contextuel pour assister à un élargissement méthodologique à même de fournir un cadre approprié à notre analyse.

À partir des années 1980, on assiste en effet à une ouverture progressive de la perspective sémiotique, jusque-là confinée à l'analyse de structures statiques et closes, à des aspects culturels, anthropologiques et sociaux de la communication publicitaire. L'intérêt se porte désormais vers des éléments plus proprement contextuels de la communication marchande : références sociétales et temporelles des messages, allusions intertextuelles, perspectives d'interprétation, contextes de production et structures médiatiques. Dans ce cadre se déploie notamment une approche qui ouvrira de nouvelles perspectives prometteuses à l'enseignement de la langue-culture étrangère : l'approche sociosémiotique. Envisageant les discours publicitaires comme le résultat non seulement d'un processus créateur de significations, mais aussi comme un espace où sont générés des phénomènes de société, cette approche permet d'étudier la publicité à la fois comme le reflet et le lieu de construction et de transformation d'une réalité sociale et culturelle (De Iulio 2016, 29-30). En dynamisant la conception autrefois plus figée de la publicité comme « miroir statique » (Cathelat 2001, 270), la nouvelle approche permet d'étudier le discours publicitaire comme un objet en mouvement, d'éclairer la genèse et les transformations de ce discours en tenant compte des contextes dans lesquels il s'inscrit, des acteurs sociaux et culturels mis en scène. L'analyse sociosémiotique de la publicité se présente ainsi comme une approche fort prometteuse pour notre propos en ce qu'elle permet d'établir des « cartographies » signifiantes, de saisir des « univers de sens (...) qui arrivent à s'affirmer comme étant objectifs et indiscutables car ils coïncident avec le répertoire de croyances et convictions partagées par les récepteurs » (De Iulio 2016, 31). Dans une telle perspective, la publicité se présente comme un livre ouvert sur la culture d'une société, un miroir à diffraction, particulièrement sensible puisqu'à l'écoute permanente des aspirations profondes de ses récepteurs, de leurs désirs et de leurs questionnements. " Au diapason de la culture d'ensemble », la communication publicitaire se confond désormais avec les valeurs que son produit manifeste et symbolise : «Sous le message de consommation se lit en filigrane le message de civilisation » (Cathelat 2001, 266).

Dans le cadre de ce tournant contextuel on voit naître aussi des études comparatives qui portent un regard interculturel sur la publicité, cherchant à saisir les particularités nationales du discours marchand et les caractéristiques propres aux sociétés dont il émane. Nombre de ces études présentent la production publicitaire française comme un phénomène 'à part', avec des caractéristiques bien spécifiques. Avant de passer à l'étude 
d'exemples concrets, il paraît à présent indispensable de cerner les particularités de ce discours publicitaire pour poser les jalons de notre analyse.

\section{3}

\section{COMMUNICATION PUBLICITAIRE : « L'EXCEPTION FRANÇAISE »}

Dans le sillage de la mondialisation de l'économie, l'attention des chercheurs se porte tout naturellement vers les spécificités culturelles et sociales des nouveaux marchés et de leurs communautés-cibles. À partir des années 1980, on voit ainsi paraître nombre d'études qui analysent les cultures publicitaires dans une perspective comparative, multidisciplinaire et interculturelle. L'intérêt se tourne d'abord vers les productions publicitaires américaine et asiatiques (notamment japonaise), ce n'est que vers le début des années 1990 que les recherches se consacreront plus proprement à la culture publicitaire française (Samiee \& Jeong 1994 ; Taylor, Hoy \& Haley 1996) ${ }^{2}$. Dans le courant de cette décennie, de nombreuses recherches, américaines et allemandes notamment, se pencheront sur le phénomène et en identifieront les traits saillants ${ }^{3}$. Dès lors, la culture publicitaire française sera perçue et étudiée comme un phénomène particulier, une 'exception culturelle'.

On peut faire remonter les origines de cette 'exception' aux années 1960, décennie lors de laquelle la publicité, par le développement des médias-audio-visuels, étend considérablement son influence. Cette époque est marquée par l'expansion, en Europe, des multinationales anglo-saxonnes qui tentent de conquérir les marchés avec des approches marketing testées sur des millions de consommateurs américains. Sous leur influence, la France passe très rapidement à une nouvelle forme de publicité dite 'scientifique', mettant en avant les avantages objectifs des produits et services pour convaincre les consommateurs par des arguments comparatifs et chiffrés. Écoutons à ce propos le témoignage d'un ancien publicitaire :

Seuls les arguments objectifs devaient être retenus dans notre démarche. Ensuite, ils devaient être assenés clairement et d'une façon répétitive à nos 'cibles' ménagères (...) Il n'était pas question de rêve dans nos annonces mais de comparaisons objectives et banales. (Bernard Brochand, Préface de Cathelat 2001)

La réaction à cet apport venu d'outre-Atlantique ne se fait pas attendre : cette expérience donne lieu à un « vaste mouvement irréversible conduisant de la publicité

2 Selon un inventaire des publications sur la publicité interculturelle parues entre 1980 et 1992 dans les plus grandes revues de marketing américaines, seules quatre des 25 études portent spécifiquement sur la France (Samiee \& Jeong 1994).

3 Les Français eux-mêmes ne se sont guère penchés sur la spécificité de leur culture publicitaire : «The French are somewhat reluctant to analyze their own work (...) There's a feeling among French creatives that if you analyze something, you destroy its power, you destroy the power of creation » (Taylor, Hoy \& Haley 1996, 11). 
'scientifique, quantitative, objective et banale' à une communication 'qualitative, imaginaire, artistique et spectaculaire' » (ibid.). Ceux qu'on appelle les 'Nouveaux publicitaires ${ }^{4}$ transformeront radicalement la communication marchande tout au long des prochaines décennies, rejetant le systématique et le répétitif pour se tourner vers l'intuition et le rêve. Bien davantage que leurs prédécesseurs, ils se nourriront de 'l'air du temps', du patrimoine culturel français dont leurs créations n'auront cesse de reproduire et de déployer les motifs. «L'acte créatif publicitaire se transforme en prenant en compte des tendances et des aspirations de groupes et de sous-groupes (...), (leurs) valeurs sociales, morales et culturelles. » (ibid.) Aucun pays occidental n'a vécu en si peu de temps un tel bouleversement dans sa communication marchande.

«La publicité est la fleur de la vie contemporaine, elle est affirmation d'optimisme et de gaieté, elle distrait l'œil et l'esprit » (Blaise Cendrars, cité dans Leduc 1969, XXIII). Cette définition, formulée dans le contexte de ce tournant créatif, résume avec éloquence la caractéristique la plus remarquable sans doute du discours publicitaire français. Les études qui se sont penchées sur le phénomène s'accordent à reconnaître la prépondérance du critère esthétique : «On a pu constater qu'en France la forme, c'est-à-dire la façon dont une information est présentée, joue un rôle au moins aussi important que le fond du message » (Schroeder 1994, 35). Davantage que dans d'autres cultures, la publicité française entretient avec son public un rapport de séduction; avant même de présenter les caractéristiques d'un produit ou d'un service, il s'agit de créer un cadre, une ambiance propre à aguicher le consommateur : « The cultural belief that the role of advertising is not to convey information is so strong that when French advertising is most factual, most informative, and most measurable (...) it is not considered advertising » (Taylor, Hoy \& Haley 1996, 9). Ou comme a pu l'affirmer Maurice Lévy : « le publicitairement correct est de ne pas parler du produit » (cité dans Mirande \& Le Bourdonnec 1995, 73). Pour séduire son public-cible, la publicité doit être, avant tout, visuellement attrayante, « this fits the French visual orientation to life and reflects their sensitivity to aesthetics, color, and design », précise E. T. Hall, l'un des pères fondateurs de la communication interculturelle (Hall \& Hall 1990, 127). Impossible, pour un publicitaire français, d'ignorer le design : la séduction passe par une mise en scène de la beauté, une attention toute particulière portée aux formes, aux couleurs et au style. À l'opposé de l'approche 'hard sell' (vente agressive) venue des États-Unis, laquelle consiste à employer un vocabulaire direct et à asséner au public-cible des arguments objectifs pour emporter la vente, la publicité 'à la française' se caractérise par une communication indirecte, plus subtile et plus 'douce' ('soft sell') (Taylor, Hoy \& Haley 1996). Par leurs messages délivrés en filigrane, les créateurs entretiennent avec leurs cibles un rapport interactif, ludique et complice. À cet égard, il n'est pas surprenant que le publicitaire français, davantage que ses confrères étrangers, puisse se concevoir lui-même comme un artiste, peintre et poète du quotidien :

4 Toute une série d'agences publicitaires se créent à partir de 1968. 
«A lot of creative teams see themselves like poets of daily life. That's probably an important part of the DNA of the French market » (Reeves 2017).

Cette approche 'douce' fait la part belle, aussi, à l'émotion. La publicité française a ceci de particulier qu'elle recourt avec prédilection à des arguments affectifs et sentimentaux. Cela la distingue d'autres cultures publicitaires, comme le suggère, non sans une nuance d'ironie, le célèbre publicitaire Jacques Séguéla :

Je dis toujours qu'il y a trois sortes de publicités. Les Anglais font une publicité qui vient de la tête mais touche le cœur : c'est toujours plutôt intellectuel. Les Français font une publicité qui vient du cœur et touche la tête : elle repose souvent sur une imagerie romantique, émotionnelle et sensuelle. Les Américains font une publicité qui vient de la tête et touche le porte-monnaie. (cité dans Tungate 2009, 127)

Plusieurs études comparatives menées au fil des décennies ont montré que le recours aux appels émotionnels est plus élevé en France que dans d'autres pays, occidentaux notamment (cf. par exemple Angelini \& Federico 2010 ; Taylor, Hoy \& Haley 1996 ; Biswas, Olsen \& Carlet 1992). Les émotions, dans les publicités françaises, se manifestent sous diverses formes : rêve, évasion, romantisme, association d'images et de symboles forts, ... Il est deux caractéristiques, cependant, qui davantage encore que les autres, constituent une marque spécifique du discours publicitaire français : le grand spectacle et l'amour/l'érotisme. Dans un souci de singularité, on déploie des mises en scène audacieuses et partant excentriques, on recourt avec prédilection à des cadres inédits, des décors somptueux. Comme le précisent Taylor, Hoy \& Haley (1996, 8), « Spectacle incorporates the meanings of sight, an attraction, a show, a play, a story (histoire), a lavish production, high production values $»$. Pour être efficace, le discours publicitaire doit adopter une dramaturgie qui relève de la composition théâtrale : «A good french advertisement shares the beautiful qualities of a well-made theatrical film » (ibid.).

Dans un tel contexte, il n'est pas surprenant que la romance (au sens large) occupe une place prépondérante : "Romantic appeal brings more success than anything else » (Taylor, Hoy \& Haley 1996, 8). La réputation de la France comme pays de l'amour ${ }^{5}$ se reflète et s'exprime, pour ne pas dire se multiplie dans sa culture publicitaire. Les études s'accordent à reconnaître le rôle central du discours amoureux, en se concentrant sur quelques thèmes majeurs. Sur l'image de la femme d'abord, séduisante et séductrice, fatale, sensuelle et parfois provocante, image qui depuis les années 1990 tend à se polariser vers deux extrêmes, la femme-objet et la femme-sujet ${ }^{6}$. Sur les nombreuses références

5 À ce sujet voir l'étude de Jean-Jacques Pauvert : L'Amour à la française ou l'exception étrange, parue en 1997 aux Éditions du Rocher.

6 L'évolution de l'image de la femme dans la publicité française et internationale est traitée dans de nombreux sites et blogs Internet. 
érotiques ensuite. « On a souvent reproché à la publicité française d'être trop portée sur le sexe » (Angelini \& Federico 2010, 119 ; Federico 1998, 11) : plusieurs études comparatives, d'origine américaine notamment, ont dénoncé l'esprit libertin, parfois gaulois, qui marque ce discours publicitaire (Angelini \& Federico 2010, Federico 1998, Taylor, Hoy \& Haley, 1996 ; Biswas, Olsen \& Carlet 1992). Comme le précise toutefois Michael Rothschild, la notion de 'sex-appeal', à la différence de l'acception américaine, est à prendre au sens large du mot, comme une combinaison de beauté, d'attirance physique, de nudité et d'allusion érotique (cité dans Federico 1998, 5). Les publicités françaises sont pleines de suggestions ; les symboles sexuels, à commencer par la beauté raffinée des modèles (masculins ou féminins), font appel à l'inconscient et au rêve, pour être attirants, les corps ne doivent pas être dénudés. Les sous-entendus, et c'est là notre troisième point, sont nombreux dans les publicités françaises, davantage que dans d'autres cultures publicitaires, ils invitent le public-cible à lire entre les lignes, à déceler un sens caché qui le porte, dans un élan ludique, au-delà des apparences, du message évident (Angelini \& Federico 2010 ; Federico 1998 ; Taylor, Hoy \& Haley, 1996).

Ce langage à double sens se présente aussi comme la caractéristique la plus distinctive sans doute de l'humour à la française (Angelini \& Federico 2010 ; Taylor, Hoy \& Haley, 1996 ; Biswas, Olsen \& Carlet 1992). La France, on le sait, entretient un rapport passionné avec sa langue. Faut-il s'étonner dès lors que son discours publicitaire fasse la part belle au verbe, qu'elle regorge de jeux de mots, d'associations surprenantes et drôles ? La créativité verbale se déploie notamment dans les slogans publicitaires, où l'attention du récepteur est attirée par des énigmes, des calembours, des effets sonores et rythmiques, allitérations, assonances, tautogrammes, virelangues ou trompe-oreille (Damamme-Gilbert 1998). La langue publicitaire apparaît comme un terrain propice à l'innovation lexicale ; les linguistes qui se sont penchés sur le phénomène n'ont cessé de souligner sa créativité au point qu'Adam et Bonhomme en font l'un des moteurs de l'argumentation publicitaire $(1997,158)$. Dans une culture marquée par une la forte tradition linguistico-normative, ces transgressions innovatives, cependant, se heurtent aussi à une résistance 'puriste' (cf. par exemple Vicari 2016 et Bonhomme 2002) ; dans ce sens, force est de souligner que l'humour publicitaire ne fait pas - loin de là - l'unanimité auprès de son public-cible français.

Une dernière caractéristique, enfin, la plus fructueuse sans doute pour notre propos didactique, est la tendance de ce discours publicitaire à pratiquer un certain 'narcissisme culturel" : "French advertising continually makes reference to French culture rather than just simply to the product itself. » (Angelini \& Federico 2010, 120). L'univers de référence pour le consommateur français est presque toujours celui de la France : ses régions, son histoire, son art, sa littérature, le caractère des gens qui y vivent et leur mentalité (ibid.). Pour reprendre les termes de Mark Tungate, «Les Français ont la réputation d'être nationalistes et un tantinet chauvins » $(2009,127)$. Ce 'narcissisme' se reflète dans une prédilection marquée pour des sujets invoquant la 'grandeur' de la France, son passé 
glorieux, son rayonnement et son faste. À cet égard, le prestige de la marque Paris, perçue comme la capitale mondiale du romantisme, de l'art de vivre, du luxe et de la culture, constitue un motif central, pour ne pas dire une pierre angulaire de ce discours marchand. Même si ces dernières années, cette image a été fortement ébranlée par des événements politiques et sociaux.

Penchons-nous à présent sur la campagne actuelle d'une grande compagnie française pour tenter d'y repérer les éléments caractéristiques de cette 'exception française'.

\section{MISE EN PRATIQUE : AIR FRANCE - FRANCE IS IN THE AIR}

Pour introduire les apprenants FLE à l'étude de la 'culture française', rien de tel que de la leur faire découvrir par le biais de publicités actuelles. À ce propos, la dernière campagne d'Air France, dont le slogan - «France is in the Air » - représente à lui seul tout un programme, constitue un sujet abondant qui se prête parfaitement à une analyse culturelle. Dans les prochains paragraphes, nous chercherons à mettre en œuvre les considérations théoriques esquissées dans les pages précédentes en nous penchant sur les 'artefacts' de cette campagne publicitaire.

Développée par l'agence de publicité BETC, la campagne « France is in the Air 》 est lancée en 2014, dans le cadre d'un plan de restructuration stratégique. L'objectif est de faire monter en gamme la compagnie, de dynamiser la marque à l'international pour lui permettre de faire face à une concurrence toujours plus acharnée dans le secteur aérien. Si elle s'articule - et c'est là une première pour la compagnie - autour d'un slogan en langue anglaise, la nouvelle campagne place la France au cœur de sa stratégie : «C'est une campagne sur la francité, c'est l'anti french bashing par excellence de façon assumée, avec la répétition du mot 'France' dans l'accroche » (Véronique Gohmann, citée dans Les Échos, en ligne). Déclinée en une série de visuels illustrant les produits proposés par Air France (le confort de l'A380, les nouvelles classes Affaires et Première, la gastronomie, le réseau, le programme SkyPriority) et les destinations emblématiques de la compagnie, la campagne est diffusée sur trois canaux : en print (affiches), vidéo et digital.

« La force de la compagnie, ce sont ses racines, l'esprit français. Quand vous mettez un pied à bord d'un avion, vous êtes déjà un peu en France ", note Tiphaine du Plessis, l'une des directrices de la campagne (citée dans Roosen 2015). Dans une période de forte concurrence dans l'univers aérien, la nouvelle stratégie de la compagnie consiste à élever la marque en promettant au client de " goûter à l'art de vivre et à la French Touch en voyageant avec Air France » (Les Échos, en ligne). À cet effet, on a voulu créer « une forme de connivence et de proximité avec le public », précise la direction de la communication d'Air France (Challenges, en ligne). Pour se rendre accessible au plus grand nombre de cibles possibles, la compagnie met clairement l'accent sur des valeurs portées par la France à l'international, déclinées dans des visuels et des slogans aisés à déchiffrer 
pour tous les publics. C'est par le biais de ces messages 'évidents' que nous entamerons notre analyse culturelle.

La campagne «France is in the Air » regorge de motifs du patrimoine culturel et du savoir-faire de la France aisément repérables pour les apprenants de FLE. On y découvre des allusions à de grands sujets historiques : Louis XIV (l'enfant-roi, Versailles et les jardins de Le Nôtre), Marie-Antoinette dans son boudoir, la Révolution (la Marianne installée dans une chaise à porteurs sous le titre "Un confort révolutionnaire »). Au divertissement à la française (Moulin Rouge et French cancan, petits rats de l'Opéra). À la gastronomie (petits fours présentés sur un éventail de fourchettes); à la haute couture (une robe évasée en forme de Tour Eiffel). La campagne multiplie aussi les clins d'œil aux stéréotypes sur la France et les Français : marinières, bérets basques, grenouilles (une femme en costume de batracien sous le titre « Oh les jambes joyeuses »), vin rouge et 'French kiss'. Très répandus dans les publicités internationales jusqu'au tournant du millénaire, ces stéréotypes trouvent depuis peu un relais dans les publicités nationales, où ils se transforment et se modernisent en s'imprégnant d'une ironie subtile et souvent caricaturale (Culture Pub, en ligne). Dans le cas d'Air France, ils sont mis en scène dans un esprit à la fois raffiné et festif. La comparaison de ces stéréotypes dans les publicités de différents pays permettra aux apprenants de saisir la spécificité de l'approche française, de faire ressortir la référentialité ludique de son discours marchand.

La campagne d'Air France se prête parfaitement à une analyse culturelle en ce qu'elle recèle toutes les caractéristiques de 'l'exception française' telle que nous l'avons définie plus haut. D'abord et surtout par son 'narcissisme culturel'. Dans cette campagne, la France se célèbre elle-même en se présentant à son public-cible sous un jour prestigieux - stratégie qui se veut le reflet d'une perception internationale : "La France est plus que jamais chargée en valeurs positives », comme l'explique Caroline Fontaine, responsable de la marque et de la publicité chez Air France (citée dans Roosen 2015). « Ambassadrice » de son pays, la compagnie aérienne promeut les valeurs d'une France idéalisée et mythique, celle de la 'grande' histoire, des traditions et du luxe. Cette stratégie, qui proclame l'ouverture au monde tout en mettant en avant le patrimoine et des valeurs nationales, n'est pas sans présenter, à première vue, une contradiction inhérente. Elle traduit de fait un désir d'affirmation de soi en proclamant la pérennité des valeurs portées par la France. C'est ainsi que la campagne fait la part belle au 'grand spectacle', par des mises en scènes raffinées qui s'inscrivent dans des cadres somptueux. Le château de Versailles, notamment, qui constitue le motif de plusieurs visuels - sans jamais être nommément cité ni désigné par l'image. Les scénarios se déroulent dans le parc du château, ses allées dérobées ( Un confort révolutionnaire »), le labyrinthe de Le Nôtre (une longue traîne rouge évoquant le fil d'Ariane dans un dédale de destinations, sous le titre « Ciel, me voilà ! »). On se penchera notamment sur l'affiche intitulée « Son Altesse A380 », dont la composition fait bien ressortir toute la grandiloquence de cette campagne (figure 1). On y voit un Airbus atterrir sur l'allée centrale du parc de Versailles transformée en tarmac : 


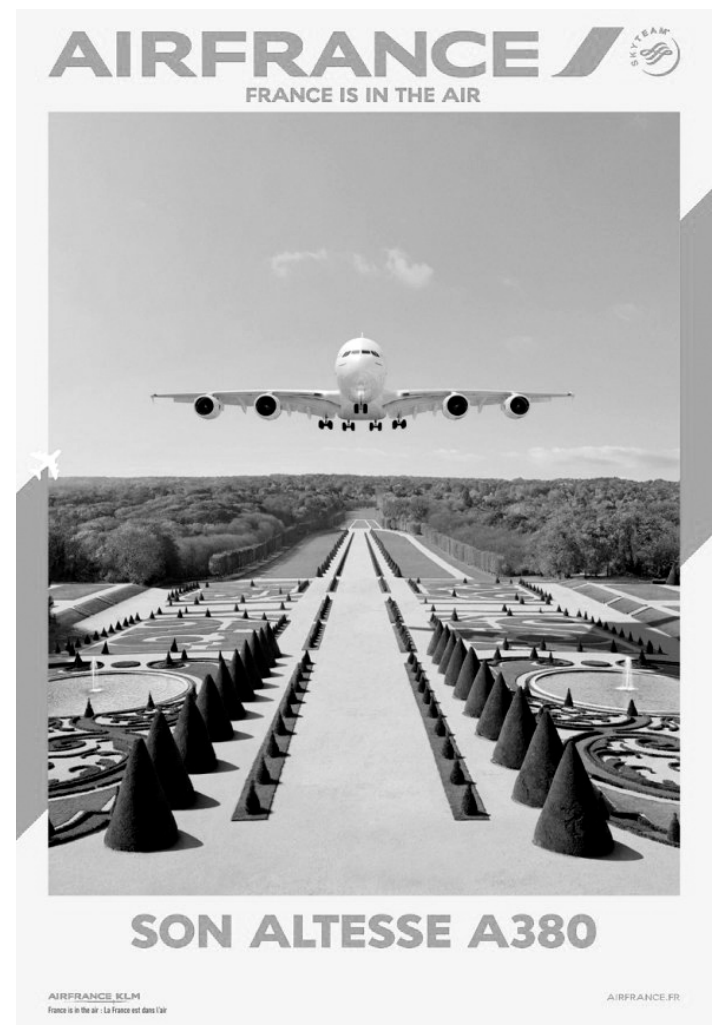

Figure 1 : Son Altesse A380

http://www.refletcommunication.com/blog/print/air-france-france-is-in-the-air/ (retrouvé le 20.06.2019) (C) Air France http://corporate.airfrance.com

Le titre est évocateur : on y promeut le plus gros avion de tous les temps en l'associant, par une double métonymie et une substitution métaphorique, au Roi-Soleil et à son gigantesque palais. Cette notion de grandeur associée au luxe fastueux de l'espace trouve de nombreux échos dans toute la campagne d'Air France, particulièrement dans le spot publicitaire «France is in the Air », où des groupes de personnes se balancent, à grands mouvements, dans un vaste espace intérieur (cabine sans sièges) bordé de colonnades évoquant le classicisme de Versailles.

Peu soucieuses de déployer des arguments raisonnés, ces mises en scène improbables et surréalistes reflètent un parti pris d'esthétique au détriment d'une communication plus rationnelle, par exemple sur les avantages 'standard' que pourrait offrir cette compagnie aérienne (sécurité, fiabilité, prix). "France is in the Air » se caractérise par une quête d'extravagance et de beauté qui se décline à la fois dans des scénarios inédits et un visuel percutant. Visuel à la fois moderne et traditionnel en ce qu'il présente des combinaisons -insolites et surprenantes - de couleurs fortes où prédomine la tricolore 
française, tout en faisant référence au passé d'affichiste de renom de la compagnie. Voire à des œuvres d'art emblématiques, comme l'Olympia de Manet, dont le motif est repris et détourné dans plusieurs affiches pour faire ressortir le confort du voyage (par exemple avec Marie-Antoinette dans son boudoir et la Marianne confortablement installée dans son siège à porteurs, figures 3 et 4 ).

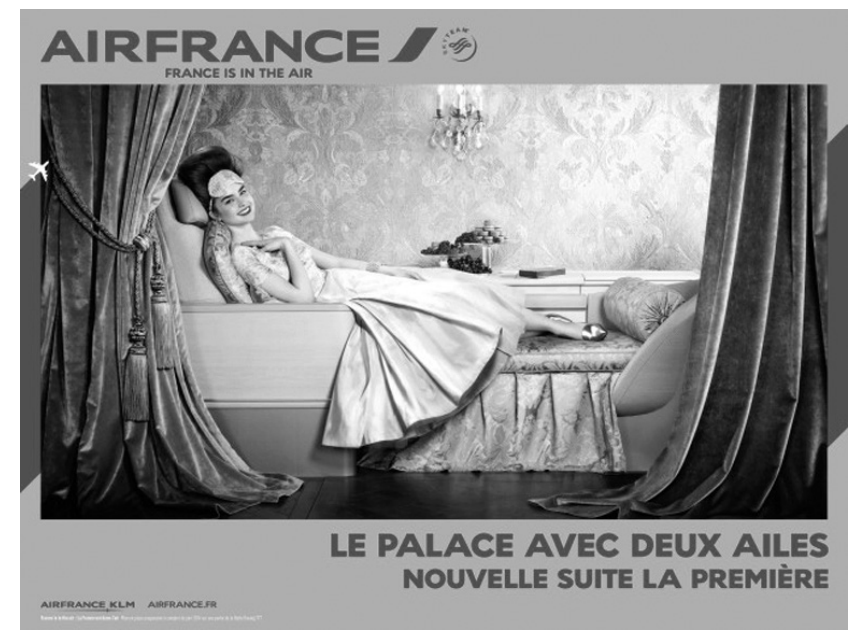

Figure 2 : Le palace avec deux ailes

http://www.refletcommunication.com/blog/print/air-france-france-is-in-the-air/ (retrouvé le 20.06.2019) (C) Air France http://corporate.airfrance.com

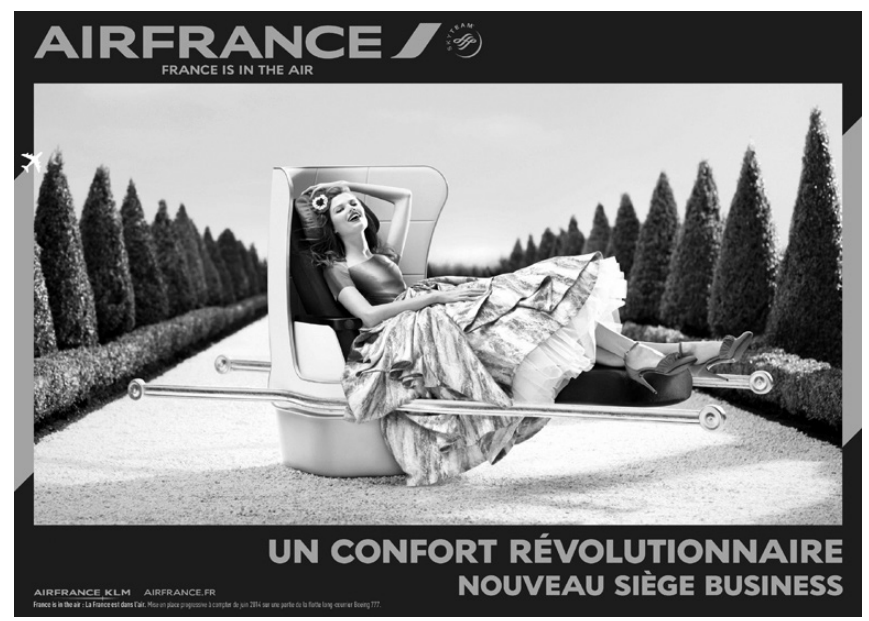

Figure 3 : Un confort révolutionnaire

http://www.refletcommunication.com/blog/print/air-france-france-is-in-the-air/ (retrouvé le 20.06.2019) (C) Air France http://corporate.airfrance.com 
À ce sujet, on soulignera toutefois la retenue pudique d'Air France : le personnage n'est jamais dénudé dans ses visuels. Si elle reflète l'aspiration de la compagnie à se situer sur le haut de gamme, l'attention portée à la forme, à l'esthétique des arguments traduit aussi et surtout la caractéristique bien française d'une communication publicitaire soucieuse, nous l'avons vu, de raffinement et d'émotion. Par-delà ses ambitions marchandes, c'est un art de vivre que proclame cette campagne en insistant sur le plaisir du voyage, de ses préliminaires (des affiches qui font rêver) bien davantage que sur des arguments objectifs et marchands.

Cet art de vivre, cette émotion trouvent leur plus belle expression sans doute dans l'évocation du sentiment amoureux qui constitue, il fallait s'y attendre, l'un des thèmes majeurs de cette campagne. L'amour, dans " France is in the Air », se manifeste sous différentes formes, plus ou moins explicites. Il s'affiche clairement dans le spot de la campagne sous la forme d'un 'French kiss' échangé entre deux mouvements de balançoire - aboutissement et point culminant du film dont le thème musical « Love is in the Air », répété en boucle, se confond avec le slogan de la compagnie ${ }^{7}$. Il se profile en filigrane dans de nombreux motifs, revêtant systématiquement le visage de la séduction féminine. Représentées dans différents contextes et situations, les protagonistes constituent toujours le point focal (et souvent le seul motif) de l'image, mises en vedette par une position originale, une tenue recherchée, parfois extravagante, un maquillage haut en couleur. Elles adressent un regard soutenu au spectateur comme une invitation à ne plus détourner les yeux, dans des postures parfois suggestives, jamais vulgaires ni dégradantes. Dans cette campagne somme toute assez pudique, la séduction s'incarne dans une combinaison de beauté, d'attirance physique et d'allusion érotique, mais jamais dans la nudité. Il est très frappant que les visuels ne mettent pratiquement pas en scène de protagonistes masculins - même le Tour de France est représenté par des femmes (spot « France is in the Air »). Les rares hommes que l'on y découvre interviennent en tant que faire-valoir romantiques («Cinéma mon amour », «Et clic ! Je lui prends un billet!»), de figurants (passagers anonymes, pararazzi, ...) ou sous la forme de présences métaphoriques (Louis $\mathrm{XIV}$, les grands chefs étoilés). Une telle démarche n'est pas le seul reflet d'une 'exception culturelle', elle relève aussi d'un choix stratégique : « Air France est une Femme ! C'est ce qui ressort de toutes les études réalisées » explique Florence Bellisson, directrice de création chez BETC (citée dans De Blanzy 2014). Si la femme tient une place prépondérante dans cette campagne, présentée comme une personnalité tout aussi séduisante qu'affirmée, c'est qu'elle y incarne une compagnie soucieuse de donner d'elle-même une image moderne et forte, « très en phase avec son époque » (ibid.).

Cette campagne, qui invite en permanence son lecteur à lire entre les lignes, à déchiffrer des allusions cachées au-delà du sens patent, révèle finalement un esprit ludique,

7 Signalons que la compagnie Air France est actuellement poursuivie en Australie pour avoir prétendument violé les droits d'auteur sur la chanson de John Paul Young « Love Is In The Air» datant de 1977. 
un humour qui correspond bien aux définitions de la recherche en matière de culture publicitaire. Nombreux sont les messages qui jouent sur le double sens, par le biais de renvois thématiques, sémantiques ou formels. La campagne, nous l'avons vu, regorge de clins d'œil enjoués au patrimoine culturel de la France dont elle détourne les motifs à des fins gentiment parodiques : le Versailles de Louis XIV, la Révolution, la haute gastronomie... De par leur composition, les visuels se présentent, pour la plupart, comme des énigmes culturelles plus ou moins élaborées, dont les slogans, parfois, viennent prolonger ou compléter le champ sémantique. Slogans parsemés de jeux de mots divers : polysémies significatives («Ciel, me voilà ! »; « Ciel ! un chef »), équivoques (« Le palace à deux ailes »), métaphores (« Un réseau ébouriffant »), rimes redoublées (« Appli, ma belle appli, les yeux fermés je te suis »), allitérations ( "Cinéma mon amour »), palilogies ( «3,2,1 décollez. Oui oui oui deals »). Sans toutefois proposer des créations trop audacieuses : on ne relève aucun néologisme dans cette campagne. En fin de compte, il fallait s'y attendre, le texte n'occupe pas une place prépondérante dans ces publicités adressées à des clientèles internationales; il passe au second plan, relégué au service de l'image.

Quel bilan sociosémiotique tirer de cette analyse culturelle ? En tant que « représentation parlée du monde » (Barthes 1963), à quelle(s) réalité(s) cette campagne nous renvoie-t-elle ? Pour être efficace, la communication publicitaire, nous l'avons vu, doit se mettre au diapason des attentes collectives, prendre appui sur des mythes partagés par son public-cible, des 'archétypes' enfouis dans son inconscient commun. Prise dans la tourmente de la concurrence internationale, Air France a choisi de communiquer sur des valeurs lisibles et identifiables tout autour du monde, valeurs dont la complexité et la richesse se trouvent par là même réduites, étriquées parfois en clichés sur la France. L'image que veut transmettre cette campagne s'avère à première vue fort éloignée de la réalité du pays. De fait, plutôt que dans les messages évidents, c'est dans la forme, les arguments et les allusions cachées que l'on cherchera à identifier la « représentation parlée » de cette culture. De par son esthétique vigoureuse, ses mises en scène élaborées, c'est un esprit que reflète cette campagne, esprit soucieux de séduire ses publics-cibles par des compositions marquantes, de frapper leur attention par des scénarios et des visuels inédits. En déclinant un savoir-faire esthétique à plusieurs niveaux, Air France renvoie une représentation proprement idéalisée de la nation dont elle se veut l'ambassadrice, déployant les codes culturels et artistiques fameux et si chers au pays. " France is in the Air » a ceci de particulier que tout en promouvant l'image de la France à l'étranger, elle se fraie un accès, aussi, à ses cibles nationales en leur présentant, pour l'identification motivante, une image de soi conforme à un idéal collectif. Par-delà sa finalité marchande, la fonction de la publicité, comme le souligne Bernard Cathelat, est d'offrir à son audience « un style de pensée et de vie, un ensemble de valeurs désirables, donc motivantes, et acceptables à la fois, donc repérables, rassurantes, conformistes » (Cathelat 2001, 267). En ces temps de crise, la campagne d'Air France fournit au public-cible français un modèle symbolique chargé de satisfaction suggérée, une part de rêve, échappatoire vers le l'idéal d'une 
France atemporelle et mythique, débarrassée de ses doutes et de ses conflits. Une France sublimée et anachronique, magnifiée par son triomphe culturel, par le rayonnement de sa civilisation. Une représentation en porte-à-faux avec les réalités actuelles du pays.

\section{CONCLUSION}

Dans un monde où les particularismes nationaux sont progressivement grignotés par la logique de l'économie internationale, on peut, bien sûr, s'interroger sur le bien-fondé d'une entreprise qui vise à cerner la spécificité culturelle d'une nation en se penchant sur sa communication marchande. Il ne fait aucun doute, pourtant, que l'étude de productions publicitaires ouvre des pistes séduisantes pour sensibiliser des apprenants de FLE à l'altérité culturelle. Par l'inventaire des thèmes exploités d'abord, mais aussi par l'analyse d'un style, d'une tonalité spécifique, elle permet de faire ressortir des différences significatives, de dégager des caractéristiques propres à une culture donnée. Et de déceler, en filigrane des productions, l'image qu'une société conçoit d'elle-même - ou du moins les indices qu'elle nous en livre. Dans le cas de la campagne d'Air France, nous avons affaire à une représentation ambivalente, proclamant l'ouverture et la modernité de la France au monde tout en invoquant son passé glorieux et ses traditions célèbres. Représentation idéalisée qui cherche à affirmer la pérennité des valeurs portées par la France ainsi que leur rayonnement de par le monde contemporain. Une telle pratique, qui consiste à renvoyer à son public-cible une image 'typisée' de sa culture, correspond assurément à celle de toute démarche publicitaire, telle que la conçoit l'approche sociosémiotique : « la publicité reflète la réalité sociale, une réalité qui n'est pas un diagnostic objectif, mais une typification de ce que les individus pensent et comment ils se représentent le monde » (Sacriste 2001, 493). Ce que nous fait miroiter la campagne " France is in the Air » est, avant tout, la réalité d'une nation en quête d'identité et de sens, éternellement nostalgique de sa grandeur passée.

De telles observations, il va de soi, demandent à être prolongées, affinées, confirmées ou infirmées par l'étude d'autres campagnes publicitaires. 


\section{BIBLIOGRAPHIE}

ADAM, Jean-Michel/Marc BONHOMME (1997) L'argumentation publicitaire. Rhétorique de l'éloge et de la persuasion. Paris : Nathan.

ANGELINI, Eileen M./Salvatore FEDERICO (2010) Understanding French Culture through Advertisements Networking and Technology. Global Business Languages 3, 109-120.

BARTHES, Roland (1957) Mythologies. Paris : Éditions du Seuil.

BARTHES, Roland (1963) Le message publicitaire, rêve et poésie. Communications \& Langages 7, 91-96.

BARTHES, Roland (1964) Rhétorique de l'image. Communications 4, 40-51.

BISWAS, Abhijit/Janeen E. OLSEN/Valerie CARLET (1992) A Comparison of Print Advertisements from the United States and France. Journal of Advertising XXI (4), 73-81.

BONHOMME, Marc (2002) La publicité comme laboratoire du français actuel. L'Information grammaticale 94, 33-38.

CATHELAT, Bernard (2001) Publicité et société. Paris : Éditions Payot.

CULTURE PUB (28/11/2015) L'image de la France dans les pub étrangères. En ligne. Le 5/6/2019. http://www.culturepub.fr/chapitres/l-image-de-la-france-dans-les-pubetrangeres-2015-11-28/.

DAMAMME-GILBERT, Béatrice (1998) Advertising. Encyclopedia of Contemporary French Culture. London \& New York : Routledge.

DAMOUR, Pauline (26/03/2014) Air France opte pour une communication très patriotique. Challenges. En ligne. Le 4/6/2019. https://www.challenges.fr/entreprise/ air-france-opte-pour-une-communication-tres-patriotique_160786.

DE BLANZY, Adrien (27/03/2014) Air France ou les plaisirs à la française. $L ' A D N$. En ligne. Le 12/6/2019. https://www.ladn.eu/news-business/actualites-agences/ air-france-ou-les-plaisirs-a-la-francaise/.

DE IULIO, Simona (2016) Étudier la publicité. Presses Universitaires de Grenoble.

ECO, Umberto (1972) La structure absente. Paris : Mercure de France.

FEDERICO, Salvatore (1998) Sexe et publicité à la française. Communication et langages $117,4-11$.

HALL, Edward T./Mildred R. HALL (1990) Understanding cultural differences: Germans, French and Americans. London : Nicholas Brealey.

LES ÉCHOS (16/07/2015) Air France : l'élégance et la poésie revisitées. En ligne. Le 4/6/2019. https://business.lesechos.fr/directions-marketing/communication/publicite/ air-france-l-elegance-et-la-poesie-revisitees-200798.php.

LEDUC, Robert (1969) La publicité, une force au service de l'entreprise. Paris : Dunod. MIRANDE, Louise/Yannick LE BOURDONNEC (1995) Pub : Le Rebond? Enjeux Les Échos, 72-79. 
REEVES, Alex (2017) What is it About French Advertising? The Beak Street Bugle. En ligne. Le 28/5/2019. http://beakstreetbugle.com/articles/view/566/ what-is-it-about-french-advertising.

REY, Alain et al. (2010) Dictionnaire historique de la langue française. Paris : Le Robert. ROOSEN, Mélanie (06/03/2015) France is in the Air. L'ADN. En ligne. Le 3/6/2019. https://www.ladn.eu/news-business/actualites-agences/france-is-in-the-air/.

SACRISTE, Valérie (2001) Sociologie de la communication publicitaire. L'Année sociologique 51, 487-498.

SAMIEE, Saeed/Insik JEONG (1994) Cross-cultural Research in Advertising: An Assessment of Methodologies. Journal of the Academy of Marketing Science 22 (3), 205-217.

SCHROEDER, Michael (1994) France - Allemagne: l'existence de deux logiques de communication. U. E. Koch, D. Schröter et A. Pierre (éd.), Images Médiatiques franco-allemandes. Munich : Verlag Reinhard Fischer, 21-42.

TAYLOR, Ronald E./Mariea G. HOY/Eric HALEY (1996) How French Advertising Professionals Develop Creative Strategy. Journal of Advertising XXV (1), 1-14.

TUNGATE, Mark (2009) Le Monde de la pub. Paris : Dunod.

VICARI, Stefano (2016) De l'engouement publicitaire pour la langue 'relâchée' : linguistes, non-linguistes et corpus en confrontation. Publifarum 26. En ligne. Le 31/5/2019. http://www.publifarum.farum.it/ezine_articles.php?art_id=367.

\section{POVZETEK}

Oglaševalska/-e kultura/-e pri poučevanju francoščine kot tujega jezika: »France is in the air«, analiza oglaševalske kampanje

Oglaševanje se večinoma opira na edinstvene vsebine in dogodke v obliki »artefaktov«, ki so idealni za ustvarjanje predstave o kulturni drugačnosti, obenem pa naslovnikom pomagajo prevzeti in razumeti družbene vrednote v določenem okolju. Pri pouku tujega jezika so se oglasi izkazali za izjemno uporabno sredstvo, ki učencem omogoča raziskovanje vseh vidikov, ki jih ponuja oglaševanje. Pričujoči prispevek predstavlja didaktične pristope $\mathrm{k}$ raziskovanju medkulturnosti pri pouku francoščine kot tujega jezika (FLE). Pri tem se osredotoča na oglaševalsko kampanjo družbe Air France $\mathrm{z}$ naslovom »France is in the Air«. Primerjalna analiza omenjene kampanje je pokazala, da so v kampanji predstavljeni številni vidiki »francoske izjemnosti«. Pri tem kampanja s pridom izkorišča naslednje prvine oglaševanja: vzbujanje najrazličnejših čustev (sanje, pobeg, romantičnost - francoski oglasi naslovnike večinoma privabljajo z zelo privlačnimi argumenti, ki vzbujajo bolj ali manj močna čustva in občutja), vizualno in jezikovno privlačna podoba ter vključevanje prvin kulturnega narcisizma, zabavljaštva (spektakel), zapeljevanja, ljubezni in humorja. Namen kampanje družbe Air France je tujcem prikazati vrednote francoskega naroda; kampanja tako 
ciljnemu občinstvu simbolično slika izmuzljivi ideal poveličevane, mitične in brezčasne Francije. $\mathrm{S}$ socio-semantičnega vidika kampanja slika francoski narod v iskanju lastne identitete in pomena, obenem pa je v njej mogoče zaznati tudi večno nostalgijo po veličini pretekle dobe.

Ključne besede: francoščina kot tuji jezik (FLE), oglaševanje, marketing, medkulturna komunikacija, socio-semantična analiza, Air France

\section{ABSTRACT}

\section{Advertising culture(s) in French as a foreign language classroom: "France is in the air", analysis of a campaign}

Advertising generally relies on unique content and scenarios in the form of 'artefacts' that are ideally suited for raising awareness of cultural otherness and helping recipients to develop and understand the societal values in a particular setting. In the context of foreign language teaching, using advertisements has proven to be a particularly rich means for students to explore the full range of characteristics this phenomenon has to offer. This article presents didactic approaches to exploring the intercultural dimension in French as a foreign language (FFL) classes by focusing on the "France is in the Air" campaign of Air France. Seen from a comparative perspective, the campaign features many elements of the 'French Exception' theme to communicate various forms of emotion (dream, escape, romanticism - French advertising generally has strongly compelling affective and sentimental arguments), visual and verbal aesthetics, cultural narcissism, showmanship ('spectacle'), seduction, love, and humour. It aims to convey national values abroad and symbolically portrays the elusive ideal of a glorified, mythical and timeless France to its target audience. When seen from a socio-semantic angle, the Air France campaign suggests that, above all, the nation is searching for identity and meaning while at the same time expressing eternal nostalgia for the greatness of a bygone era.

Keywords: French as a foreign language (FFL), advertising, marketing, intercultural communication, socio-semantic analysis, Air France

\section{RÉSUMÉ}

Lieu de construction et reflet de valeurs sociétales propres au contexte dans lequel elle s'inscrit, la publicité propose des contenus et des scénarios caractéristiques, des 'artefacts' qui se prêtent idéalement à la sensibilisation pour l'altérité culturelle. Dans le cas de l'enseignement d'une langue étrangère, elle se présente comme un outil particulièrement riche pour faire découvrir aux étudiants le phénomène sous diverses facettes. Cet article présente des pistes didactiques destinées à 
explorer la dimension interculturelle en classe de FLE (français langue étrangère), en se penchant sur la dernière campagne publicitaire de la compagnie Air France intitulée «France is in the Air ». Étudiée dans une perspective comparative, cette campagne recèle de nombreux éléments caractéristiques d'une 'exception française' en matière de communication publicitaire : émotion sous diverses formes (rêve, évasion, romantisme), esthétique visuelle et verbale, narcissisme culturel, grand spectacle, séduction, amour, humour. Destinée à porter les valeurs nationales à l'étranger, elle offre à son public-cible un modèle symbolique propageant l'idéal irréel d'une France magnifiée, mythique et atemporelle. D'un point de vue sociosémantique, ce que nous fait miroiter la campagne d'Air France est, avant tout, la réalité d'une nation en quête d'identité et de sens, éternellement nostalgique de sa grandeur passée.

Mots-clés : FLE (français langue étrangère), publicité, marketing, communication interculturelle, analyse sociosémantique, Air France 